\title{
WEAKLY ALMOST PERIODIC FUNCTIONS WITH ZERO MEAN
}

\author{
BY CHING CHOU $^{1}$
}

Communicated by Kenneth A. Ross, August 30, 1973

Let $G$ be a locally compact group, $C(G)$ the space of bounded complexvalued continuous functions on $G$ with the sup norm and $U C(G)$ the closed subspace of $C(G)$ consisting of uniformly continuous functions. If $f \in C(G)$ and $x \in G$ the left \{right translation of $f$ by $x, l_{x} f\left\{r_{x} f\right\}$, is given by $\left(l_{x} f\right)(y)=f(x y)\left\{\left(r_{x} f\right)(y)=f(y x)\right\} . f \in C(G)$ is said to be weakly almost periodic (w.a.p.) if the set $\left\{l_{x} f: x \in G\right\}$ is relatively compact with respect to the weak topology of $C(G)$. The set of all w.a.p. functions on $G$, designated by $W(G)$, is a closed subalgebra of $U C(G)$ and is closed under translations. Moreover, it has a unique invariant mean $m: m \in W(G)^{*}$, $\|m\|=1, m \geqq 0$ and $m\left(l_{x} f\right)=m\left(r_{x} f\right)=m(f)$ for $x \in G$ and $f \in W(G)$. (This is the well-known Ryll-Nardzewski fixed point theorem. Burckel's monograph [1] is a convenient reference for this and other facts concerning w.a.p. functions.) Let $W_{0}(G)=\{f \in W(G): m(|f|)=0\}$. Then $W_{0}(G)$ is a closed subalgebra of $W(G)$ and $W(G)=W_{0}(G) \oplus A(G)$ where $A(G)=$ $\{f \in C(G): f$ almost periodic $\}$, cf. [1]. Therefore the study of $W(G)$ is somehow reduced to the study of $A(G)$ and $W_{0}(G)$. For almost periodic functions there are useful characterization theorems and a deep approximation theorem; cf. [4]. But little is known about functions in $W_{0}(G)$. One well-known fact is that $C_{0}(G) \subset W_{0}(G)$ if $G$ is noncompact. Here $C_{0}(G)=\{f \in C(G): f$ vanishing at infinity $\}$. Burckel [1, Theorem 4.17] proved that if $G$ is abelian and noncompact then $C_{0}(G) \subsetneq W_{0}(G)$. His proof is short but some quite deep results in harmonic analysis on abelian groups are used. He conjectured that the abelian hypothesis is inessential. Even though the abelian hypothesis can be weakened considerably, there exists a noncompact solvable group $G$ with $C_{0}(G)=W_{0}(G)$.

Let $\boldsymbol{C}$ be the set of complex numbers, $\boldsymbol{T}$ the unit circle in $\boldsymbol{C}$ and $\boldsymbol{R}$ the set of real numbers.

AMS (MOS) subject classifications (1970). Primary 22A10, 43A07, 46N05; Secondary 22D99, 43A60, 46E15.

${ }^{1}$ Supported in part by NSF grant PO 34190-001. 
THEOREM 1. Let $\Gamma=\boldsymbol{C} \times \boldsymbol{T}$ with the product topology and with multiplication given by

$$
(z, \exp (i \theta))\left(z^{\prime}, \exp \left(i \theta^{\prime}\right)\right)=\left(z+\exp (i \theta) z^{\prime}, \exp \left(i\left(\theta+\theta^{\prime}\right)\right)\right) .
$$

Then $W_{0}(\Gamma)=C_{0}(\Gamma)$.

The proof depends, among other things, on the fact that each w.a.p. function is uniformly continuous and that if $f$ is uniformly continuous on $\Gamma$, then given $\varepsilon>0$ there exists $\delta>0$ such that

$$
|f(z \exp (i \varphi), \exp (i \theta))-f(z, \exp (i \theta))|<\varepsilon
$$

if $(z, \exp (i \theta)) \in \Gamma$ and $|\exp (i \varphi)-1|<\delta$.

REMARK. In [5] deLeeuw and Glicksberg proved that if $G$ is abelian and if $H$ is a closed subgroup of $G$ then $W(H)=W(G) \mid H$. It is not hard to see that their theorem does not hold for the nonabelian solvable group $\Gamma$ and its closed normal subgroup $C$. In fact, $W(C)$ is not even contained in $U C(\Gamma) \mid C$.

For further discussions we need the following.

Definition. Let $G$ be a locally compact group. $G$ is said to have property (E) if either $G$ is compact or there exists a set $X \subset G$ such that (i) $\mathrm{cl} X$ is noncompact, (ii) given a neighborhood $U$, of the identity $e$, the set $\bigcap\left\{x U x^{-1}: x \in X \cup X^{-1}\right\}$ is again a neighborhood of $e$.

The main reason for us to consider groups with property $(\mathrm{E})$ is that we have the following: Suppose that $X$ satisfies (ii) above and that $V$ is a neighborhood of $e$ with $x V \cap x^{\prime} V=\varnothing$ if $x, x^{\prime} \in X, x \neq x^{\prime}$. Pick $h \in C(G)$ with its support contained in $V$. Then $\sum\left\{l_{x^{-1}} f: x \in X\right\} \in U C(G)$.

Example 1. Each of the following groups has property (E): (a) a group with equivalent right and left uniform structures, e.g., an abelian group or a discrete group; (b) a group with noncompact center, e.g., $\operatorname{GL}(n, \boldsymbol{R})$ or the subgroup of $\mathrm{GL}(n, \boldsymbol{R})$ consisting of upper triangular matrices $\left(x_{i j}\right)$ with $x_{i i}=1, i=1,2, \cdots, n$.

Example 2. Each of the following groups fails to have property (E): (a) $\operatorname{SL}(2, \boldsymbol{R})$, (b) the group $\Gamma$ in Theorem 1 and (c) the group $\boldsymbol{R} \times \boldsymbol{R} \times \boldsymbol{T}$ with multiplication defined by

$$
(x, y, \exp (i \theta))\left(x^{\prime}, y^{\prime}, \exp \left(i \theta^{\prime}\right)\right)=\left(x+x^{\prime}, y+y^{\prime}, \exp i\left(\theta+\theta^{\prime}+x y^{\prime}\right)\right) \text {. }
$$

THEOREM 2. Let $G$ be a noncompact locally compact group with property (E). Then there exists a linear isometry from $l^{\infty}$ into the quotient Banach space $W_{0}(G) / C_{0}(G)$.

The proof uses a generalization of Lemma 1 in [2] and some ideas in Rudin [7].

Example 2(c) tells us that a nilpotent group may fail to have property (E). But it is not hard to derive the following from Theorem 2. 
COROLlaRY. Let $G$ be a noncompact locally compact nilpotent group. Then there exists a linear isometry from $l^{\infty}$ into $W_{0}(G) / C_{0}(G)$.

By Theorem 1, we know that in the above corollary the condition "nilpotent" cannot be replaced by "solvable".

$\mu \in U C(G)^{*}$ is called a left \{right invariant mean if $\|\mu\|=1, \mu \geqq 0$ and $\mu\left(l_{x} f\right)=\mu(f)\left\{\mu\left(r_{x} f\right)=\mu(f)\right\}$ for $x \in G$ and $f \in U C(G)$. Denote the set of all left \{right\} invariant means on $U C(G)$ by $M L(G)\{M R(G)\}$. If $M L(G) \neq \varnothing$ then $G$ is said to be amenable. It is known that solvable groups and compact groups are amenable. The reader is referred to [6] for a quite complete account of the theory of amenable groups and invariant means. If $G$ is amenable it is natural to consider the space $F_{0}(G)=$ $\{f \in U C(G): \mu(|f|)=0$ as $\mu$ runs through $M L(G) \cup M R(G)\}$. It is known that $F_{0}(G) \supset W_{0}(G)$; cf. [1]. In [1, Theorem 3.19], Burckel proved that $F_{0}(\boldsymbol{R}) \supsetneqq W_{0}(\boldsymbol{R})$ where $\boldsymbol{R}$ is the additive group of reals with the usual topology. We have the following stronger results.

THEOREM 3. If $G$ is a locally compact amenable group with noncompact center, then there is a linear isometry from $l^{\infty}$ into $F_{0}(G) / W_{0}(G)$.

THEOREM 4. Let $G$ be an almost connected noncompact locally compact solvable group. Then there is a linear isometry from $l^{\infty}$ into $F_{0}(G) / W_{0}(G)$.

The ideas in [1, Theorem 3.19] are used in the proof of Theorem 3. To prove Theorem 4 it is enough to consider the special case that $G=R^{2} \times{ }_{\eta} T^{k}$, the topological semidirect product of $\boldsymbol{R}^{2}$ and $\boldsymbol{T}^{k}$ where $\eta$ is a continuous homomorphism of $\boldsymbol{T}^{k}$ into $\operatorname{GL}(2, \boldsymbol{R})$.

The results announced in this paper will be among those presented in detail in [3].

\section{REFERENCES}

1. R. B. Burckel, Weakly almost periodic functions on semigroups, Gordon and Breach, New York, 1970. MR 41 \#8562.

2. C. Chou, On the size of the set of left invariant means on a semigroup, Proc. Amer. Math. Soc. 23 (1969), 199-205. MR 40 \#710.

3. - Weakly almost periodic functions and almost convergent functions on a group (submitted).

4. C. Corduneanu, Almost periodic functions, Interscience, New York, 1968.

5. K. deLeeuw and I. Glicksberg, The decomposition of certain group representations, J. Analyse Math. 15 (1965), 135-192. MR 32 \#4211.

6. F. P. Greenleaf, Invariant means on topological groups and their applications, Van Nostrand Math. Studies no. 16, Van Nostrand-Reinhold, New York, 1969. MR 40 \#4776.

7. W. Rudin, Weak almost periodic functions and Fourier-Stieltjes transforms, Duke Math. J. 26 (1959), 215-220. MR 21 \#1492.

Department of Mathematics, State University of New York at Buffalo, AMHERST, NeW York 14226 